\title{
On Some Dynamics of a Diffusive Lotka-Volterra Competition-Advection System with Lethal Boundary Conditions
}

\author{
Dongxuan Zhao \\ College of Science, University of Shanghai for Science and Technology, Shanghai, China \\ Email: 1138533788@qq.com
}

How to cite this paper: Zhao, D.X. (2021) On Some Dynamics of a Diffusive Lotka-Volterra Competition-Advection System with Lethal Boundary Conditions. Applied Mathematics, 12, 287-297. https://doi.org/10.4236/am.2021.124020

Received: March 9, 2021

Accepted: April 16, 2021

Published: April 19, 2021

Copyright $\odot 2021$ by author(s) and Scientific Research Publishing Inc. This work is licensed under the Creative Commons Attribution International License (CC BY 4.0).

http://creativecommons.org/licenses/by/4.0/

\begin{abstract}
In this paper, we mainly study a diffusive Lotka-Volterra competition-advection system with lethal boundary conditions in a general heterogeneous environment. By using the basic theory of partial differential equations and some nonlinear analysis techniques, we investigate the existence, uniqueness and global asymptotic behavior of steady-state solutions of the system equations. The existence, uniqueness and global asymptotic behavior of steady-state solutions are proved by upper and lower solutions, maximum principle and other methods. In theory, the methods and skills to deal with this kind of nonlinear problem are further developed, which provides a theoretical basis for understanding some practical problems.
\end{abstract}

\section{Keywords}

Global Asymptotic Stability, Competing Systems, Positive Steady-State Solution, Coexistence

\section{Introduction}

In this paper, we consider the following coupled reaction-diffusion-advection system

$$
\left\{\begin{array}{l}
u_{t}=d_{1} \Delta u-\alpha_{1} \nabla \cdot(u \nabla p)+u(a(x)-b(x) u-c(x) v) \text { in } \Omega \times(0, \infty), \\
v_{t}=d_{2} \Delta v-\alpha_{2} \nabla \cdot(v \nabla p)+v(d(x)-e(x) u-f(x) v) \text { in } \Omega \times(0, \infty), \\
u=v=0 \text { on } \partial \Omega \times(0, \infty), \\
u(x, 0)=u_{0}(x), v(x, 0)=v_{0}(x) \text { in } \Omega,
\end{array}\right.
$$

and its corresponding elliptic system 


$$
\begin{cases}d_{1} \Delta u-\alpha_{1} \nabla \cdot(u \nabla p)+u(a(x)-b(x) u-c(x) v)=0 & \text { in } \Omega, \\ d_{2} \Delta v-\alpha_{2} \nabla \cdot(v \nabla p)+v(d(x)-e(x) u-f(x) v)=0 & \text { in } \Omega, \\ u=v=0 \text { on } \partial \Omega, & \end{cases}
$$

where the functions $a(x), b(x), c(x), d(x), e(x)$ and $f(x) \in C^{\alpha}(\bar{\Omega})$ are nonnegative for some $0<\alpha<1$. Here $u(x, t)$ and $v(x, t)$ represent population densities of two competing species at location $x$ and time $t$ respectively, $\Omega \subseteq \mathbf{R}^{N}$ is a bounded domain with $C^{2+\alpha}$ boundary. The boundary condition describes the situation where the boundary of $\Omega$ is lethal to the species. Here $d_{1}, d_{2}>0$ are the diffusion coefficients of species $u$ and $v$, respectively. The non-constant function $p(x) \in C^{2}(\Omega)$ is used to specify the advective direction. When the coefficients $a(x), b(x), c(x), d(x), e(x), f(x)$ are constants (homogeneous environment), the system (1.1) has been studied in the references [1]-[6] and the references therein.

Since the environments are usually heterogeneous in the real world, it is more reasonable to assume that the coefficients in the system (1.1) are nonconstant [7]. In recent years, $\mathrm{He}$ and $\mathrm{Ni}$ considered a two-species Lotka-Volterra competition-diffusion model with homogeneous Neumann boundary conditions. The effect of spatial heterogeneity and spatial homogeneity of environment on two competing species and their different competition abilities are studied (see [8] [9] [10] [11] [12] and the references therein for details). Moreover, some limiting behaviors of coexistence state are also studied in [8]. In [13], Xu and $\mathrm{Ni}$ addressed the question of the dynamics of the system for two competing species in a general heterogeneous environment with lethal boundary conditions.

Motivated by the above work, this paper aims to deal with a more general model (1.1) with advection effects, where the diffusion coefficients, advection coefficients, resource functions and competition rates are all spatially heterogeneous. Throughout this paper, we assume that

$$
\frac{\alpha_{1}}{d_{1}}=\frac{\alpha_{2}}{d_{2}}=: \eta(\text { a constant })
$$

Letting $U=u \mathrm{e}^{-\eta p}, V=v \mathrm{e}^{-\eta p}$, one can see that both Equation (1.1) and Equation (1.2) are equivalent to the following systems

$$
\begin{aligned}
& \begin{cases}U_{t}=d_{1} \Delta U+\alpha_{1} \nabla U \cdot \nabla p+U(a(x)-b(x) u-c(x) v) & \text { in } \Omega \times(0, \infty), \\
V_{t}=d_{2} \Delta V+\alpha_{2} \nabla V \cdot \nabla p+V(d(x)-e(x) u-f(x) v) & \text { in } \Omega \times(0, \infty), \\
U(x, 0)=V(x, 0)=0 \quad \text { in } \Omega, \\
U=V=0 \quad \text { on } \partial \Omega \times(0, \infty),\end{cases} \\
& \qquad \begin{cases}-d_{1} \Delta U-\alpha_{1} \nabla U \cdot \nabla p=U(a(x)-b(x) u-c(x) v) & \text { in } \Omega, \\
-d_{2} \Delta V-\alpha_{2} \nabla V \cdot \nabla p=V(d(x)-e(x) u-f(x) v) & \text { in } \Omega, \\
U=V=0 & \text { on } \partial \Omega .\end{cases}
\end{aligned}
$$

It is not hard to see from [5] that for any $A(x) \in C^{\alpha}(\bar{\Omega}), d, \alpha>0$, if $A(x)>d \lambda_{1}$ for $x \in \bar{\Omega}$, 


$$
\left\{\begin{array}{l}
d \Delta \theta-\alpha \nabla \cdot(\theta \nabla p)+\theta(A(x)-\theta)=0 \quad \text { in } \Omega \\
\theta=0 \text { on } \partial \Omega
\end{array}\right.
$$

has a unique positive solution $\theta_{d, A(x)} \in C^{2+\alpha}(\bar{\Omega})$. Here $\lambda_{1}>0$ is the principal eigenvalue of the eigenvalue problem

$$
\begin{cases}-\Delta \varphi-\eta \nabla p \cdot \nabla \varphi=\lambda_{1} \varphi & \text { in } \Omega, \\ \varphi=0 & \text { on } \partial \Omega .\end{cases}
$$

Let $\Theta_{d, A(x)}=\theta_{d, A(x)} \mathrm{e}^{-\eta p}$, then we have

$$
\left\{\begin{array}{l}
d \Delta \Theta_{d, A(x)}+\alpha \nabla p \cdot \nabla \Theta_{d, A(x)}+\Theta_{d, A(x)}\left(A(x)-\Theta_{d, A(x)} e^{\eta p}\right)=0 \text { in } \Omega, \\
\Theta_{d, A(x)}=0 \text { on } \partial \Omega .
\end{array}\right.
$$

Throughout this paper, we always assume that

$$
b(x)>0, f(x)>0, a(x)>d_{1} \lambda_{1}, d(x)>d_{2} \lambda_{1} \text { for all } x \in \bar{\Omega}
$$

and denote

$$
\bar{u}(x)=\frac{\theta_{d_{1}, a(x)}}{\min _{x \in \bar{\Omega}} b(x)}, \bar{v}(x)=\frac{\theta_{d_{2}, d(x)}}{\min _{x \in \bar{\Omega}} f(x)} .
$$

Now we state our main results for the problems (1.4) and (1.5).

Theorem 1.1. (Existence) Suppose that (1.9) holds. If $a(x), c(x), d(x), e(x), \bar{u}(x)$ and $\bar{v}(x)$ satisfy

$$
a(x)>\lambda_{1} d_{1}+c(x) \bar{v}(x), d(x)>\lambda_{1} d_{2}+e(x) \bar{u}(x),
$$

and $c(x), e(x)$ are all nonnegative or all $x \in \bar{\Omega}$, then the system (1.4) has a positive steady-state $(\tilde{U}(x), \tilde{V}(x))$.

In order to give the second theorem, we need to introduce the following problem

$$
\left\{\begin{array}{l}
d \Delta w-\alpha \nabla \cdot(w \nabla p)+w(a(x)-b(x) w)=0 \quad \text { in } \Omega, \\
w=0 \text { on } \partial \Omega .
\end{array}\right.
$$

The existence of $w$ is guaranteed by (1.11). In addition, we denote the solution of Equation (1.12) by $w_{d, \alpha, a, b}$. On the other hand, let $(H, I)$ be the solution of $\left\{\begin{array}{l}0=d_{1} \Delta H+\alpha_{1} \nabla p \cdot \nabla H+H\left[a(x)-b(x) H(x) \mathrm{e}^{\eta p}-c(x) w_{d_{2}, \alpha_{2}, d, f}\right] \text { in } \Omega, \\ 0=d_{2} \Delta I+\alpha_{2} \nabla p \cdot \nabla I+I\left[d(x)-e(x) w_{d_{1}, \alpha_{1}, a, b}-f(x) I(x) \mathrm{e}^{\eta p}\right] \text { in } \Omega, \\ H=I=0 \text { on } \partial \Omega .\end{array}\right.$

Theorem 1.2. (Uniqueness). Assume that all the hypotheses of Theorem 1.1 are satisfied. If

$$
c^{2}(x) \frac{w_{d_{1}, \alpha_{1}, a, b}}{I \mathrm{e}^{\eta p}}+2 c(x) e(x)+e(x)^{2} \frac{w_{d_{2}, \alpha_{2}, d, f}}{H \mathrm{e}^{\eta p}}<4 b(x) f(x), \quad x \in \bar{\Omega},
$$

then the steady-state $(\tilde{U}(x), \tilde{V}(x))$ of Equation (1.4) is unique.

Theorem 1.3. (Global asymptotic stability). Assume that the hypotheses of Theorem 1.2 
Are satisfied. Let $(U(x, t), V(x, t))$ be the solution of Equation (1.4) with $U(x, 0), V(x, 0) \geq$, $\equiv 0, U(x, 0), V(x, 0) \in C^{1}(\bar{\Omega})$, and vanishing on $\partial \Omega$. Then

$$
(U(x, t), V(x, t)) \rightarrow(\tilde{U}(x), \tilde{V}(x)) \text { as } t \rightarrow \infty,
$$

uniformly in $\bar{\Omega}$.

The rest of this paper is organized as follows: Theorem 1.1 and Theorem 1.2 are proved Section 2. Theorem 1.3 is established in Section 3 by proving a more general theorem.

\section{Proof of Theorem 1.1, 1.2}

Proof of Theorem 1.1:

We denote $\bar{U}=\bar{u}(x) \mathrm{e}^{-\eta p}, \bar{u}(x)=c_{1} \theta_{d_{1}, a(x)}$, where $c_{1}=\frac{1}{\min _{x \in \bar{\Omega}} b(x)}$. We choose $r_{1}>0, r_{2}>0$ small enough, and let $\underline{U}=r_{1} \varphi(x), \underline{V}=r_{2} \varphi(x)$, where $\varphi(x)$ is defined by (1.7). In fact, it follows from $1-b(x) c_{1}<0$ that

$$
\begin{aligned}
& d_{1} \Delta \bar{U}+\alpha_{1} \nabla \bar{U} \cdot \nabla p+\bar{U}\left(a(x)-b(x) \bar{u}(x)-c(x) r_{2} \varphi(x) \mathrm{e}^{\eta p}\right) \\
& =c_{1}\left(d_{1} \Delta \Theta_{d_{1}, a(x)}+\alpha_{1} \nabla p \cdot \nabla \Theta_{d_{1}, a(x)}\right) \\
& \quad+c_{1} \Theta_{d_{1}, a(x)}\left(a(x)-b(x) \bar{u}(x)-c(x) r_{2} \varphi(x) \mathrm{e}^{\eta p}\right) \\
& =c_{1} \Theta_{d_{1}, a(x)}\left[\left(1-b(x) c_{1}\right) \theta_{d_{1}, a(x)}-c(x) r_{2} \varphi(x) \mathrm{e}^{\eta p}\right] \leq 0
\end{aligned}
$$

for $r_{2}$ sufficiently small. Since $d(x)>d_{2} \lambda_{1}+e(x) \bar{u}(x)$, it then follows that

$$
d_{2} \Delta\left(r_{2} \varphi(x)\right)+\alpha_{2} \nabla p \cdot \nabla\left(r_{2} \varphi(x)\right)+r_{2} \varphi(x)\left(d(x)-f(x) r_{2} \varphi(x) \mathrm{e}^{\eta p}-e(x) \bar{u}(x)\right)
$$$$
=r_{2}\left(d_{2} \Delta \varphi(x)+\alpha_{2} \nabla p \cdot \nabla \varphi(x)\right)+r_{2} \varphi(x)\left(d(x)-f(x) r_{2} \varphi(x) \mathrm{e}^{\eta p}-c_{1} e(x) \theta_{d_{1}, a(x)}\right)
$$$$
=-r_{2} d_{2} \lambda_{1} \varphi(x)+r_{2} \varphi(x)\left(d(x)-c_{1} e(x) \theta_{d_{1}, a(x)}-f(x) r_{2} \varphi(x) \mathrm{e}^{\eta p}\right)
$$

$=r_{2} \varphi(x)\left(-d_{2} \lambda_{1}+d(x)-c_{1} e(x) \theta_{d_{1}, a(x)}-f(x) r_{2} \varphi(x) \mathrm{e}^{\eta p}\right) \geq 0$,

for small $r_{2}$. Therefore $\left(\bar{U}, r_{2} \varphi(x)\right)$ is an upper solution of Equation (1.5).

Similarly, by letting $c_{2}=\frac{1}{\min _{x \in \bar{\Omega}} f(x)}, \bar{V}=\bar{v}(x) \mathrm{e}^{-\eta p}, \bar{v}(x)=c_{2} \theta_{d_{2}, d(x)}$, we have that $\left(r_{1} \varphi(x), \bar{V}\right)$ is a lower solution of Equation (1.5) provided that $1-f(x) c_{2}<0, a(x)>\lambda_{1} d_{1}+c(x) \bar{v}(x)$, for $r_{1}$ sufficiently small.

Hence, Equation (1.4) has a positive steady-state $(\tilde{U}(x), \tilde{V}(x))$ by the method of upper and lower solution [11].

Next, we will prove the uniqueness.

Proof of Theorem 1.2:

Suppose that $\left(\tilde{U}_{1}(x), \tilde{V}_{1}(x)\right),\left(\tilde{U}_{2}(x), \tilde{V}_{2}(x)\right)$ are two strictly positive steady-state of the system (1.4). We denote

$\tilde{u}_{1}(x)=\tilde{U}_{1}(x) \mathrm{e}^{\eta p}, \tilde{u}_{2}(x)=\tilde{U}_{2}(x) \mathrm{e}^{\eta p}, \tilde{v}_{1}(x)=\tilde{V}_{1}(x) \mathrm{e}^{\eta p}$ and $\tilde{V}_{2}(x)=\tilde{V}_{2}(x) \mathrm{e}^{\eta p}$.

Now let

$$
\left\{\begin{array}{l}
M(x)=\tilde{U}_{1}(x)-\tilde{U}_{2}(x), \\
N(x)=\tilde{V}_{1}(x)-\tilde{V}_{2}(x) .
\end{array}\right.
$$


Notice that

$$
\left\{\begin{array}{l}
d_{1} \Delta \tilde{U}_{1}(x)+\alpha_{1} \nabla p \cdot \nabla \tilde{U}_{1}(x)+\tilde{U}_{1}\left(a(x)-b(x) \tilde{u}_{1}(x)-c \tilde{v}_{1}(x)\right)=0 \\
d_{1} \Delta \tilde{U}_{2}(x)+\alpha_{1} \nabla p \cdot \nabla \tilde{U}_{2}(x)+\tilde{U}_{2}\left(a(x)-b(x) \tilde{u}_{2}(x)-c \tilde{v}_{2}(x)\right)=0 .
\end{array}\right.
$$

By subtracting the above two equations, we obtain that

$$
\begin{aligned}
& d_{1} \Delta M(x)+\alpha_{1} \nabla p \cdot \nabla M(x)+M(x) a(x)-b(x) \tilde{U}_{1}(x) \tilde{u}_{1}(x) \\
& +b(x) \tilde{U}_{2}(x) \tilde{u}_{2}(x)-c(x) \tilde{U}_{1}(x) \tilde{V}_{1}(x)+c(x) \tilde{U}_{2}(x) \tilde{V}_{2}(x) \\
& =d_{1} \Delta M(x)+\alpha_{1} \nabla p \cdot \nabla M(x)+M(x) a(x)+b(x)\left(\tilde{U}_{2}(x) \tilde{u}_{2}(x)\right. \\
& \left.-\tilde{U}_{1}(x) \tilde{u}_{1}(x)\right)+c(x)\left(\tilde{U}_{2}(x) \tilde{V}_{2}(x)-\tilde{U}_{1}(x) \tilde{v}_{1}(x)\right) \\
& =d_{1} \Delta M(x)+\alpha_{1} \nabla p \cdot \nabla M(x)+M(x) a(x)-b(x) M(x) \mathrm{e}^{\eta p}\left(\tilde{U}_{1}(x)+\tilde{U}_{2}(x)\right) \\
& +c(x) \mathrm{e}^{\eta p}\left(\tilde{U}_{2}(x) \tilde{V}_{2}(x)-\tilde{U}_{1}(x) \tilde{V}_{1}(x)+\tilde{U}_{2}(x) \tilde{V}_{1}(x)-\tilde{U}_{2}(x) \tilde{V}_{1}(x)\right) \\
& =d_{1} \Delta M(x)+\alpha_{1} \nabla p \cdot \nabla M(x)+M(x) a(x)-b(x) M(x) \mathrm{e}^{\eta p}\left(\tilde{U}_{1}(x)+\tilde{U}_{2}(x)\right) \\
& -c(x) M(x) \mathrm{e}^{\eta p} \tilde{V}_{1}(x)-c(x) N(x) \mathrm{e}^{\eta p} \tilde{U}_{2}(x) \\
& =0 .
\end{aligned}
$$

By means of the similar arguments above, we have

$$
\begin{aligned}
& d_{2} \Delta N(x)+\alpha_{2} \nabla p \cdot \nabla N(x)+N(x) d(x)-e(x) \tilde{V}_{1}(x) \tilde{u}_{1}(x) \\
& +e(x) \tilde{V}_{2}(x) \tilde{u}_{2}(x)-f(x) \tilde{V}_{1}(x) \tilde{V}_{1}(x)+f(x) \tilde{V}_{2}(x) \tilde{V}_{2}(x) \\
& =d_{2} \Delta N(x)+\alpha_{2} \nabla p \cdot \nabla N(x)+N(x) d(x)-f(x) N(x) \mathrm{e}^{\eta p}\left(\tilde{V}_{1}(x)+\tilde{V}_{2}(x)\right) \\
& -e(x) N(x) \mathrm{e}^{\eta p} \tilde{U}_{2}(x)-e(x) M(x) \mathrm{e}^{\eta p} \tilde{V}_{1}(x) \\
& =0 .
\end{aligned}
$$

Therefore, we obtain that

$$
\left\{\begin{array}{l}
d_{1} \Delta M+\alpha_{1} \nabla p \cdot \nabla M+\left[a(x)-b(x) \tilde{u}_{1}(x)-c(x) \tilde{v}_{1}(x)\right] M(x)-I_{1}=0 \text { in } \Omega, \\
d_{2} \Delta N+\alpha_{2} \nabla p \cdot \nabla N+\left[d(x)-e(x) \tilde{u}_{2}(x)-f(x) \tilde{v}_{2}(x)\right] N(x)-I_{2}=0 \text { in } \Omega, \\
M(x)=0, N(x)=0 \text { on } \partial \Omega,
\end{array}\right.
$$

where

$$
\left\{\begin{array}{l}
I_{1}=M(x) b(x) \mathrm{e}^{\eta p} \tilde{U}_{2}(x)+N(x) c(x) \mathrm{e}^{\eta p} \tilde{U}_{2}(x), \\
I_{2}=N(x) f(x) \mathrm{e}^{\eta p} \tilde{V}_{1}(x)+M(x) e(x) \mathrm{e}^{\eta p} \tilde{V}_{1}(x)
\end{array}\right.
$$

That is

$$
\left\{\begin{array}{l}
I_{1}=M(x) b(x) \tilde{u}_{2}(x)+N(x) c(x) \tilde{u}_{2}(x), \\
I_{2}=M(x) e(x) \tilde{v}_{1}(x)+N(x) f(x) \tilde{v}_{1}(x) .
\end{array}\right.
$$

Now, we introduce an eigenvalue problem

$$
\left\{\begin{array}{l}
d_{1} \Delta \varphi+\alpha_{1} \nabla p \cdot \nabla \varphi+\left(a(x)-b(x) \tilde{u}_{1}(x)-c(x) \tilde{v}_{1}\right) \varphi+\sigma \varphi=0 \text { in } \Omega, \\
\varphi=0 \quad \text { on } \partial \Omega
\end{array}\right.
$$

where the principal eigenvalue is given by 


$$
\sigma=\inf _{\varphi \in H_{0}^{1}(\Omega)} \frac{d_{1} \int_{\Omega}|\nabla \varphi|^{2} \mathrm{~d} x-\int \alpha_{1} \nabla p \cdot \nabla \varphi \varphi \mathrm{d} x-\int_{\Omega}\left(a(x)-b(x) \tilde{u}_{1}(x)-c(x) \tilde{v}_{1}(x)\right) \varphi^{2} \mathrm{~d} x}{\int_{\Omega} \varphi^{2} \mathrm{~d} x} .
$$

Since $\tilde{U}_{1}(x)$ is a positive solution

$$
\left\{\begin{array}{l}
d_{1} \Delta \varphi+\alpha_{1} \nabla p \cdot \nabla \varphi+\left(a(x)-b(x) \tilde{u}_{1}(x)-c(x) \tilde{v}_{1}\right) \varphi=0 \text { in } \Omega, \\
\varphi=0 \text { on } \partial \Omega,
\end{array}\right.
$$

0 must be the principal eigenvalue. By the variational properties, we have

$$
0 \leq \frac{d_{1} \int_{\Omega} \mathrm{e}^{\eta p}|\nabla z|^{2} \mathrm{~d} x-\int_{\Omega} \alpha_{1} \nabla p \cdot \nabla z z \mathrm{~d} x-\int_{\Omega}\left(a(x)-b(x) \tilde{u}_{1}(x)-c(x) \tilde{v}_{1}(x)\right) \mathrm{e}^{\eta p} z^{2} \mathrm{~d} x}{\int_{\Omega} \mathrm{e}^{\eta p} z^{2} \mathrm{~d} x},
$$

and

$0 \leq \int_{\Omega} z\left[-d_{1} \nabla \cdot\left(e^{\eta p} \nabla z\right)-\alpha_{1} \nabla p \cdot \nabla z-\left(a(x)-b(x) \tilde{u}_{1}(x)-c(x) \tilde{v}_{1}(x)\right) \mathrm{e}^{\eta p} z\right] \mathrm{d} x$,

for any $z \in C^{2}(\bar{\Omega})$ which vanishes on $\partial \Omega$. Similarly, $\tilde{V}_{2}(x)$ is also a strictly positive solution of

$$
\left\{\begin{array}{l}
d_{2} \Delta \varphi+\alpha_{2} \nabla p \cdot \nabla \varphi+\left(d(x)-e(x) \tilde{u}_{2}(x)-f(x) \tilde{v}_{2}(x)\right) \varphi=0 \text { in } \Omega \\
\varphi=0 \quad \text { on } \partial \Omega
\end{array}\right.
$$

Hence, we obtain that

$$
0 \leq \int_{\Omega} z\left[-d_{2} \nabla \cdot\left(\mathrm{e}^{\eta p} \nabla z\right)-\alpha_{2} \nabla p \cdot \nabla z-\left(d(x)-e(x) \tilde{u}_{2}(x)-f(x) \tilde{v}_{2}(x)\right) \mathrm{e}^{\eta p} z\right] \mathrm{d} x,(2 .
$$

for any $z \in C^{2}(\bar{\Omega})$ which vanishes on $\partial \Omega$. Multiplying the first equation in (2.7) by $-\mathrm{e}^{\eta p} M(x)$, the second one by $-\mathrm{e}^{\eta p} N(x)$, integrating over $\Omega$, it then follows from (2.14) and (2.16) that

$$
\begin{aligned}
& \int_{\Omega} \mathrm{e}^{\eta p}\left(M(x)^{2} b(x) \tilde{u}_{2}(x)+N(x) M(x) c(x) \tilde{u}_{2}(x)\right. \\
& \left.+M(x) N(x) e(x) \tilde{v}_{1}(x)+N(x)^{2} f(x) \tilde{v}_{1}(x)\right) \mathrm{d} x \leq 0 .
\end{aligned}
$$

By comparison principle, we obtain that

$$
\tilde{u}_{1}(x)<w_{d_{1}, \alpha_{1}, a, b},
$$

where $w_{d_{1}, \alpha_{1}, a, b}$ is defined in Equation (1.12). Similarly, it follows that

$$
\tilde{v}_{1}(x)<w_{d_{2}, \alpha_{2}, d, f} .
$$

Therefore, we can obtain that

$$
\tilde{u}_{1}(x)>H \mathrm{e}^{\eta p}, \quad \tilde{v}_{1}(x)>I \mathrm{e}^{\eta p},
$$

where $H, I$ satisfy Equation (1.13). Similarly, there hold

$$
\left\{\begin{array}{l}
\tilde{u}_{2}(x)<w_{d_{1}, \alpha_{1}, a, b}, \quad \tilde{v}_{2}(x)<w_{d_{2}, \alpha_{2}, d, f}, \\
\tilde{u}_{2}(x)>\mathrm{He}^{\eta p}, \quad \tilde{v}_{2}(x)>I \mathrm{e}^{\eta p} .
\end{array}\right.
$$

Since it follows from (1.14) (2.18) (2.19) (2.20) and (2.21) that 


$$
\begin{aligned}
& c(x)^{2} \frac{\tilde{u}_{2}(x)}{\tilde{v}_{1}(x)}+2 c(x) e(x)+e(x)^{2} \frac{\tilde{v}_{1}(x)}{\tilde{u}_{2}(x)} \\
& <c^{2}(x) \frac{w_{d_{1}, \alpha_{1}, a, b}}{I \mathrm{e}^{\eta p}}+2 c(x) e(x)+e(x)^{2} \frac{w_{d_{2}, \alpha_{2}, d, f}}{H \mathrm{e}^{\eta p}} \\
& <4 b(x) f(x) \text { in } \Omega,
\end{aligned}
$$

it is easy to see that the quadratic expression in the integrand of (2.17) is positive definite for each $x \in \Omega$. Therefore $M(x)=0, N(x)=0$, and $\left(\tilde{U}_{1}(x), \tilde{V}_{1}(x)\right)=\left(\tilde{U}_{2}(x), \tilde{V}_{2}(x)\right)$. The proof is finished.

\section{Proof of Theorem 1.3}

Now we are in a position to prove Theorem 1.3. By Theorem 1.2 and the assumptions of Theorem 1.3, problem (1.4) has a unique positive solution $(\tilde{U}(x), \tilde{V}(x))$. Now we prove the following theorem without the assumption of (1.14) which can establish Theorem 1.3.

Theorem 3.1. Assume that the hypotheses of Theorem 1.1 are satisfied, and problem (1.5) has a unique positive solution $(\tilde{U}(x), \tilde{V}(x))$ in $\Omega$. Then $(\tilde{U}(x), \tilde{V}(x))$ is globally asymptotically stable in the following sense. Let $(U(x, t), U(x, t))$ be the solution of problem (1.4) with $U(x, 0), V(x, 0) \geq 0, \neq 0, U(x, 0), V(x, 0) \in C^{1}(\bar{\Omega})$, and vanishing on $\partial \Omega$. Then

$$
(U(x, t), V(x, t)) \rightarrow(\tilde{U}(x), \tilde{V}(x)) \text { as } t \rightarrow \infty,
$$

uniformly in $\bar{\Omega}$.

Proof. For convenience, we introduce the following notation: if $w \in C^{1}(\bar{\Omega}), w(x)>0$ for all $x \in \Omega$, and $\partial w / \partial v<0$ everywhere on $\partial \Omega$, we write $w \gg 0$. If $w, z \in C^{1}(\bar{\Omega})$, we write $w \ll z$, when $z-w \gg 0$. We first prove the theorem under the condition

$$
U(x, 0), V(x, 0) \in C^{1}(\bar{\Omega}), U(x, 0), V(x, 0) \gg 0,
$$

and

$$
U(x, 0) \leq \bar{U}(x), \quad V(x, 0) \leq \bar{V}(x),
$$

for all $x \in \bar{\Omega}$, where

$$
\bar{U}(x)=\frac{\Theta_{d_{1}, a(x)}}{\min _{x \in \bar{\Omega}} b(x)}, \bar{V}(x)=\frac{\Theta_{d_{2}, d(x)}}{\min _{x \in \bar{\Omega}} f(x)} .
$$

Let $\varphi_{1}(x)$ be the principal eigenfunction of (1.7). Choose $\varepsilon>0$ small such that

$$
\left\{\begin{array}{l}
\varepsilon \varphi_{1}(x) \leq U(x, 0), \varepsilon \varphi_{1}(x) \leq V(x, 0), \\
a(x)>d_{1} \lambda_{1}+c(x) \bar{v}(x)+b(x) \varepsilon \varphi_{1}(x) \mathrm{e}^{\eta p}, \\
d(x)>d_{2} \lambda_{1}+e(x) \bar{u}(x)+f(x) \varepsilon \varphi_{1}(x) \mathrm{e}^{\eta p} .
\end{array}\right.
$$

If we let

$$
\underline{U}=\varepsilon \varphi_{1}(x), \underline{V}=\varepsilon \varphi_{1}(x),
$$


then

$$
\left\{\begin{array}{l}
-d_{1} \Delta \bar{U}-\alpha_{1} \nabla p \cdot \nabla \bar{U} \geq \bar{U}\left(a(x)-b(x) \bar{u}(x)-c(x) \varepsilon \varphi_{1}(x) \mathrm{e}^{\eta p}\right) \text { in } \Omega, \\
-d_{1} \Delta\left(\varepsilon \varphi_{1}(x)\right)-\alpha_{1} \nabla p \cdot \nabla\left(\varepsilon \varphi_{1}(x)\right) \\
\leq \varepsilon \varphi_{1}(x)\left(a(x)-b(x) \varepsilon \varphi(x) \mathrm{e}^{\eta p}-c(x) \bar{v}(x)\right) \text { in } \Omega, \\
-d_{2} \Delta \bar{V}-\alpha_{2} \nabla p \cdot \nabla \bar{V} \geq \bar{V}\left(d(x)-e(x) \varepsilon \varphi_{1}(x) \mathrm{e}^{\eta p}-f(x) \bar{v}(x)\right) \text { in } \Omega, \\
-d_{2} \Delta\left(\varepsilon \varphi_{1}(x)\right)-\alpha_{2} \nabla p \cdot \nabla\left(\varepsilon \varphi_{1}(x)\right) \\
\leq \varepsilon \varphi_{1}(x)\left(d(x)-e(x) \bar{u}(x)-f(x) \varepsilon \varphi_{1}(x) \mathrm{e}^{\eta p}\right) \text { in } \Omega, \\
\varepsilon \varphi_{1}(x)=\bar{U}=\bar{V}=0 \quad \text { on } \partial \Omega .
\end{array}\right.
$$

By means of ([2], Theorem 1.3), the conclusion of this theorem follow from the uniqueness assumption and the inequalities

$$
\underline{U}(x, t) \leq U(x, 0) \leq \bar{U}(x, t), \underline{V}(x, t) \leq V(x, 0) \leq \bar{V}(x, t), x \in \bar{\Omega} .
$$

Next, we remove condition (3.3) from the initial data $U(x, 0), V(x, 0)$. Notice that there exists large $K>1$, such that

$$
U(x, 0)<K \bar{U}(x), \quad V(x, 0)<K \bar{V}(x),
$$

in $\Omega$. Define that $\overline{\mathbf{U}}(x, t)$ is the solution of problem

$$
\left\{\begin{array}{l}
\overline{\mathbf{U}}_{t}=d_{1} \Delta \overline{\mathbf{U}}+\alpha_{1} \nabla \overline{\mathbf{U}} \cdot \nabla p+\overline{\mathbf{U}}\left(a(x)-b(x) \overline{\mathbf{U}} \mathrm{e}^{\eta p}\right) \text { in } \Omega \times(0, \infty), \\
\overline{\mathbf{U}}(x, 0)=K \bar{U}(x) \text { in } \Omega, \\
\overline{\mathbf{U}}=0 \text { on } \partial \Omega \times(0, \infty) .
\end{array}\right.
$$

It is clear that $\overline{\mathbf{U}}$ is non-negative in $\Omega \times(0, \infty)$ and

$$
\lim _{t \rightarrow \infty} \overline{\mathbf{U}}(x, t)=\overline{\mathbf{U}}^{*}(x) \text { for } x \in \bar{\Omega},
$$

where $\overline{\mathbf{U}}^{*}(x)$ is the unique positive solution of the problem

$$
\left\{\begin{array}{l}
d_{1} \Delta \mathbf{E}+\alpha_{1} \nabla \mathbf{E} \cdot \nabla p+\mathbf{E}\left(a(x)-b(x) \mathbf{E e}^{\eta p}\right)=0 \quad \text { in } \Omega, \\
\mathbf{E}=0 \text { on } \partial \Omega .
\end{array}\right.
$$

Moreover, $\overline{\mathbf{U}}_{t}(x, 0)<0$, since $\overline{\mathbf{U}}(x, 0)$ satisfies

$$
\begin{aligned}
& d_{1} \Delta \overline{\mathbf{U}}(x, 0)+\alpha_{1} \nabla \overline{\mathbf{U}}(x, 0) \cdot \nabla p+\overline{\mathbf{U}}(x, 0)\left(a(x)-b(x) \overline{\mathbf{U}} \mathrm{e}^{\eta p}\right) \\
= & d_{1} K \Delta \bar{U}+K \alpha_{1} \nabla \bar{U} \cdot \nabla p+K \bar{U}\left(a(x)-b(x) K \bar{U} \mathrm{e}^{\eta p}\right) \\
= & K\left[d_{1} \Delta \bar{U}+\alpha_{1} \nabla \bar{U} \cdot \nabla p+\bar{U}\left(a(x)-b(x) K \bar{U} \mathrm{e}^{\eta p}\right)\right] \\
= & K\left[d_{1} \Delta \frac{\Theta_{d_{1}, a(x)}}{\min _{x \in \bar{\Omega}} b(x)}+\alpha_{1} \nabla p \cdot \nabla \frac{\Theta_{d_{1}, a(x)}}{\min _{x \in \bar{\Omega}} b(x)}\right. \\
& \left.+\frac{\Theta_{d_{1}, a(x)}}{\min _{x \in \bar{\Omega}} b(x)}\left(a(x)-b(x) K \frac{\Theta_{d_{1}, a(x)}}{\min _{x \in \bar{\Omega}} b(x)} \mathrm{e}^{\eta p}\right)\right] \\
= & \frac{K}{\min _{x \in \bar{\Omega}} b(x)}\left[\Theta_{d_{1}, a(x)}\left(\theta_{d_{1}, a(x)}-K b(x) \frac{\theta_{d_{1}, a(x)}}{\min _{x \in \bar{\Omega}} b(x)}\right)\right]<0 .
\end{aligned}
$$

By using $W^{2, p}$ estimates and Sobolev embedding, the convergence in Equation 
(3.10) is also in $C^{1}(\bar{\Omega})$ norm. Similarly, $\overline{\mathbf{V}}$ is non-negative in $\Omega \times(0, \infty)$,

$$
\lim _{t \rightarrow \infty} \overline{\mathbf{V}}(x, t)=\overline{\mathbf{V}}^{*}(x) \text { for } x \in \bar{\Omega},
$$

where $\overline{\mathbf{V}}^{*}(x)$ is the unique positive solution of the problem

$$
\left\{\begin{array}{l}
d_{2} \Delta \mathbf{F}+\alpha_{2} \nabla \mathbf{F} \cdot \nabla p+\mathbf{F}\left(d(x)-f(x) \mathbf{F} \mathrm{e}^{\eta p}\right)=0 \text { in } \Omega, \\
\mathbf{F}=0 \text { on } \partial \Omega .
\end{array}\right.
$$

On the other hand, $\overline{\mathbf{V}}_{t}(x, 0)<0$, since $\overline{\mathbf{V}}(x, 0)$ satisfies

$$
\begin{aligned}
d_{2} \Delta \overline{\mathbf{V}}(x, 0)+\alpha_{2} \nabla \overline{\mathbf{V}}(x, 0) \cdot \nabla p+\overline{\mathbf{V}}(x, 0)\left(d(x)-f(x) \overline{\mathbf{V}} \mathrm{e}^{\eta p}\right) \\
=d_{2} K \Delta \bar{V}+\alpha_{2} K \nabla \bar{V} \cdot \nabla p+K \bar{V}\left(d(x)-f(x) K \bar{V} \mathrm{e}^{\eta p}\right) \\
=K\left[d_{2} \Delta \frac{\Theta_{d_{2}, d(x)}}{\min _{x \in \bar{\Omega}} f(x)}+\alpha_{2} \nabla p \cdot \nabla \frac{\Theta_{d_{2}, d(x)}}{\min _{x \in \bar{\Omega}} f(x)}\right. \\
\left.+\frac{\Theta_{d_{2}, d(x)}}{\min _{x \in \bar{\Omega}} f(x)}\left(d(x)-f(x) K \frac{\Theta_{d_{2}, d(x)}}{\min _{x \in \bar{\Omega}} f(x)} \mathrm{e}^{\eta p}\right)\right] \\
=\frac{K}{\min _{x \in \bar{\Omega}} f(x)}\left[\Theta_{d_{2}, d(x)}\left(\theta_{d_{2}, d(x)}-K f(x) \frac{\theta_{d_{2}, d(x)}}{\min _{x \in \bar{\Omega}} f(x)}\right)\right]<0 .
\end{aligned}
$$

Let $\underline{\mathbf{U}}=\underline{\mathbf{V}}=\underline{\mathbf{u}}=\underline{\mathbf{v}} \equiv 0, \overline{\mathbf{u}}=\overline{\mathbf{U}} \mathrm{e}^{\eta p}, \overline{\mathbf{v}}=\overline{\mathbf{V}} \mathrm{e}^{\eta p}$. Then we have

$$
\left\{\begin{array}{l}
-d_{1} \Delta \overline{\mathbf{U}}-\alpha_{1} \nabla p \cdot \nabla \overline{\mathbf{U}} \geq \overline{\mathbf{U}}(a(x)-b(x) \overline{\mathbf{u}}-c(x) \underline{\mathbf{v}}), \\
-d_{2} \Delta \overline{\mathbf{V}}-\alpha_{2} \nabla p \cdot \nabla \overline{\mathbf{V}} \geq \overline{\mathbf{V}}(d(x)-e(x) \underline{\mathbf{u}}-f(x) \overline{\mathbf{v}}),
\end{array}\right.
$$

and

$$
\left\{\begin{array}{l}
0=\underline{\mathbf{U}}(x, 0) \leq U(x, 0)<\overline{\mathbf{U}}(x, 0)=K \bar{U}(x), \\
0=\underline{\mathbf{V}}(x, 0) \leq V(x, 0)<\overline{\mathbf{V}}(x, 0)=K \bar{V}(x),
\end{array}\right.
$$

for $(x, t) \in \Omega$. From (3.12), we obtain that

$$
\left\{\begin{array}{l}
\overline{\mathbf{U}}_{t}(x, 0)<0 \text { in } \Omega, \\
\left.\overline{\mathbf{U}}_{t}\right|_{\partial \Omega}=0, \\
\left(\overline{\mathbf{U}}_{t}\right)_{t}=d_{1} \Delta \overline{\mathbf{U}}_{t}+\alpha_{1} \nabla \overline{\mathbf{U}}_{t} \cdot \nabla p-2 b(x) \overline{\mathbf{U}}_{t} \overline{\mathbf{u}}_{t}+a(x) \overline{\mathbf{U}}_{t} \text { in } \Omega \times(0, \infty) .
\end{array}\right.
$$

Thus $\overline{\mathbf{U}}_{t}<0$. Similarly, one can use a similar argument as above to get $\overline{\mathbf{V}}_{t}<0$. We can also see that,

$$
\overline{\mathbf{U}}(x, 0)-U(x, 0)=K \bar{U}(x)-U(x, 0)>0,
$$

and

$$
\begin{aligned}
& (\overline{\mathbf{U}}-U)_{t}-d \Delta(\overline{\mathbf{U}}-U)-\alpha \nabla p \cdot \nabla(\overline{\mathbf{U}}-U) \\
& =\overline{\mathbf{U}}(a-b \overline{\mathbf{u}})-U(a-b u-c v) \\
& >\overline{\mathbf{U}}(a-b \overline{\mathbf{u}})-U(a-b u) \\
& =(\overline{\mathbf{U}}-U)(a-b \overline{\mathbf{u}})-b U(\overline{\mathbf{u}}-u) .
\end{aligned}
$$

Therefore $\overline{\mathbf{U}}>U$ by the comparison principle. Similarly, we obtain that $\overline{\mathbf{v}}>V$. 
We claim that $\bar{U}(x)=\frac{\Theta_{d_{1}, a(x)}}{\min _{x \in \bar{\Omega}} b(x)}$ is a strict upper solution of the problem. Equation (3.11).

In fact,

$$
\begin{aligned}
& d_{1} \Delta \bar{U}+\alpha \nabla \bar{U} \cdot \nabla p+\bar{U}\left(a(x)-b(x) \bar{U} \mathrm{e}^{\eta p}\right) \\
& =\frac{1}{\min _{x \in \bar{\Omega}} b(x)}\left[d_{1} \Delta \Theta_{d_{1}, a(x)}+\alpha \nabla \Theta_{d_{1}, a(x)} \cdot \nabla p+\Theta_{d_{1}, a(x)}(a(x)-b(x) \bar{u}(x))\right] \\
& =\frac{1}{\min _{x \in \bar{\Omega}} b(x)}\left[-\Theta_{d_{1}, a(x)}\left(a(x)-\theta_{d_{1}, a(x)}\right)+\Theta_{d_{1}, a(x)}(a(x)-b(x) \bar{u}(x))\right] \\
& =\frac{1}{\min _{x \in \bar{\Omega}} b(x)}\left[\Theta_{d_{1}, a(x)}\left(\theta_{d_{1}, a(x)}-b(x) \frac{\theta_{d_{1}, a(x)}}{\min _{x \in \bar{\Omega}} b(x)}\right)\right]<0 .
\end{aligned}
$$

By means of the similar arguments, we obtain that $\bar{V}(x)=\frac{\Theta_{d_{1}, d(x)}}{\min _{x \in \bar{\Omega}} f(x)}$ is a strict upper solution of the problem (3.14), that is

$$
d_{1} \Delta \bar{V}+\alpha_{2} \nabla \bar{V} \cdot \nabla p+\bar{V}\left(d(x)-f(x) \bar{V}(x) \mathrm{e}^{\eta p}\right)<0 .
$$

Therefore

$$
U<\overline{\mathbf{U}}, \overline{\mathbf{U}}^{*}<\bar{U}, V<\overline{\mathbf{V}}, \overline{\mathbf{V}}^{*}<\bar{V} .
$$

For $s>0, x \in \bar{\Omega}, U^{s}=U(x, s), V^{s}=V(x, s)$. From (3.10) (3.13) (3.23), we can get

$$
U^{s}(x)<\bar{U}, V^{s}(x)<\bar{V}
$$

On the other hand for $s>0$, we deduce from the theory of parabolic equations and the strong maximum principle that

$$
U^{s} \gg 0, V^{s} \gg 0 \text {. }
$$

Combined with (3.2), (3.24), (3.25), the conclusion of this theorem can be proved by using the first part of the proof.

\section{Conclusion}

In this paper, the first part constructs the system equations and expounds the theorems to be studied. The second part mainly proves the existence and uniqueness of the stable solutions of the system equations by the methods of upper and lower solutions and the maximum principle. In the third part, based on the establishment of Theorem 1.1 and 1.2, it further proves that Theorem 3.1 obtains the global asymptotic property of the steady-state solution.

\section{Conflicts of Interest}

The author declares no conflicts of interest regarding the publication of this paper.

\section{References}

[1] Consner, C. and Laser, C. (1984) Stable Coexistence States in the Volterra-Lotka 
Competition Model with Diffusion. SIAM Journal on Applied Mathematics, 44, 1112-1132. https://doi.org/10.1137/0144080

[2] Dancer, E. (1991) On the Existence and Uniqueness of Positive Solutions for Competing Species Models with Diffusion. Transactions of the American Mathematical Society, 36, 829-859. https://doi.org/10.1090/S0002-9947-1991-1028757-9

[3] Dancer, E. and Guo, Z. (1994) Uniqueness and Stability for Solutions of Competing Species Equations with Large Interactions. Communications on Applied Nonlinear Analysis, 1, 19-45.

[4] Leung, A. (2009) Nonlinear Systems of Partial Differential Equations, Applications to Life and Physical Sciences. World Scientific, Singapore.

https://doi.org/10.1142/7353

[5] Leung, A. (1989) Systems of Nonlinear partial Equations, Applications to Biology and Engineering. Kluwer Academic, New York.

[6] Ruan, W. and Pao, C. (1995) Positive Steady-State Solutions of Competing Reaction Diffusion Systems. Journal of Differential Equations, 117, 411-427.

https://doi.org/10.1006/jdeq.1995.1059

[7] Cantrell, R.S. and Cosner, C. (2003) Spatial Ecology via Reaction-Diffusion Equation, Series in Mathematical and Computational Biology. Wiley, Chichester. https://doi.org/10.1002/0470871296

[8] He, X.-Q. and Ni, W.-M. (2013) The Effects of Diffusion and Spatial Variation in Volterra-Lotka Competition-Diffusion System I: Heterogeneity vs. Homogeneity. Journal of Differential Equations, 254, 528-546. https://doi.org/10.1016/j.jde.2012.08.032

[9] He, X.-Q. and Ni, W.-M. (2013) The Effects of Diffusion and Spatial Variation in Loka-Volterra Competition-Diffusion System II: The General Case. Journal of Differential Equations, 254, 4088-4108. https://doi.org/10.1016/j.jde.2013.02.009

[10] He, X.-Q. and Ni, W.-M. (2016) Global Dynamics of the Lotka-Volterra Competition-Diffusion System: Diffusion and Spatial Heterogeneity I. Communications on Pure and Applied Mathematics, 69, 981-1014. https://doi.org/10.1002/cpa.21596

[11] He, X.-Q. and Ni, W.-M. (2016) Global Dynamics of the Lotka-Volterra Competition-Diffusion System with Equal Amount of Total Resources, II. Calculus of Variations and Partial Differential Equations, 55, Article Number: 25. https://doi.org/10.1007/s00526-016-0964-0

[12] He, X.-Q. and Ni, W.-M. (2017) Global Dynamics of the Lotka-Volterra Competition-Diffusion System with Equal Amount of Total Resources, III. Calculus of Variations and Partial Differential Equations, 56, 1-26. https://doi.org/10.1007/s00526-017-1234-5

[13] Xu, B.L. and Ni, Z.Z. (2015) Dynamics of Competing Systems in General Heterogeneous Environments. Boundary Value Problems, 2015, Article Number: 110. https://doi.org/10.1186/s13661-015-0368-7 